\title{
Multidimensional Channel Intelligent Supervision Model
}

\author{
Chun Zhou ${ }^{1, a}$, Hao Zhang ${ }^{1, b}$, Ying $X^{2}{ }^{2}$, Zhanwei Xing ${ }^{1}$ and Yin Liu ${ }^{1, c,{ }^{*}}$ \\ ${ }^{1}$ Beijing GuoDianTong Network Technology Co., Ltd.,Beijing 100070,China; \\ ${ }^{2}$ State Grid Zhejiang Province Electric Power Corporation,Hangzhou Zhejiang 310000,China. \\ a15036878 @qq.com, b276074747 @qq.com c, ${ }^{*}$ zaysaa@163.com
}

\begin{abstract}
With the gradual development of the smart grid business, business volume of the each State Grid E-Channel would be more and more large, operation complexity and daily supervision difficulty would increase, the existing E-Channel supervision mode facing the pressure is increasing. How to integrate existing resources to improve customer satisfaction index is an urgent problem to be solved in the each State Grid E-Channel. Based on user perception level and application service level, this paper uses artificial intelligence and data mining technology to analyze user perception data and operation-maintenance monitoring index data on various electronic channels which belong to State Grid Corporation of China. And multidimensional heterogeneous electronic channel service regulatory model is constructed in order to improve quality of service and customer satisfaction.
\end{abstract}

Keywords: Artificial Intelligence,E-Channel Supervision,Application Oriented Monitoring,User Perception.

\section{Introduction}

With the rapid development of modern information technology such as artificial intelligence, big data, and Internet of Things, the widespread use of mobile Internet and intelligent electronic devices, under the "Internet +" environment,the State Grid has built 95598 intelligent interactive websites and "power at hand" APP, "Electronic e" APP, State Grid Mall, "e charging" APP electronic marketing channels [1]. The number of customers of State Grid's e-marketing channels is still increasing, ensuring that the safe, efficient and stable operation of multiple marketing channel services brings new challenges and opportunities to the operation and maintenance work. The traditional operation and maintenance supervision mode has been difficult to apply to today's service and data explosion growth environment. It has the following shortcomings: First, there is a lack of application-oriented user-aware measurement mechanism, and application-oriented user-aware evaluation can focus business management on application quality. The customer satisfaction is improved by improving the application quality; secondly, the intelligent operation and maintenance supervision of the application service is lacking, and the intelligent supervision of the operation and maintenance situation can timely detect faults and abnormalities, and adjust the operation strategy by running analysis to improve the operation efficiency. This paper studies the application-oriented model of user-aware application and the operation and maintenance supervision model of applicationoriented services, and builds a multi-dimensional heterogeneous electronic channel service supervision model.

The main goal of Artificial Intelligence (AI) research is to enable machines to perform complex tasks that require human intelligence. In the 2017 government work report, Premier Li Keqiang also published artificial intelligence for the first time in the national government work report. Since Google's AlphaGo defeated Korean professional chess player Li Shishi, the AI era has arrived. Many well-known companies in the world have already carried out research on AI technology. In many fields, IBM's Watson has begun to assist humans in making some decision-making decisions and Tesla's cars to realize automatic driving technology. In order to better improve customer satisfaction and improve service quality, AI technology is used in the multi-dimensional heterogeneous electronic channel service supervision model.

Multi-dimensional heterogeneous electronic channel service supervision should be supervised from both user and application services. At the customer level, the user perception of each electronic marketing channel is monitored, and the resources corresponding to the application are monitored at the application service layer. The features extracted by manual are very fixed and limited. Therefore, 
artificial intelligence technology [2] and data mining technology are needed to deeply analyze user perception data and application service data, and build intelligent knowledge base to achieve fault prevention and rapid fault processing,with the purpose of user satisfaction improvement.

\section{User-Aware Application Supervision Model}

\subsection{Model Design.}

User perception [3,4] application supervision model consists of front-end application, background data management, analysis display and control, as shown in Figure 1. Logically, the parts work together in a low-coupling manner, and can be expanded in both horizontal and vertical directions to meet the growing demands of services and data.

The front-end application part should include 95598 intelligent interactive website, "power at hand" APP, "Electronic e" APP, State Grid Mall, "e charging" APP, national network mall and other application platforms to provide information sources for user perception and supervision. The acquisition part includes two types: active acquisition mode and monitoring mode. Active acquisition mode: From the user's point of view, the simulated user performs real user behavior at the application layer, initiates active testing, and automatically collects relevant data of the test service; monitors the collection mode: from the perspective of the operator, tests the user at the application layer through the background. The usage of the user records the relevant data of the user's various services, providing effective data support for future big data analysis, targeted product performance optimization, and in-depth understanding of the user's experience. The data part provides support for large data operations such as storage, management, and data association for a large number of structured and unstructured data generated by the acquisition layer, which facilitates the analysis layer to acquire data quickly and accurately. While the analysis and display part provides the intelligent analysis of user-aware data by providing data mining technology, the operation of displaying the perceived quality and operation optimization can intuitively supervise the user's perceived data, and let the user perceive the data as a treasure for insight into the market and consumer behavior trends. . The main function of the control part is to perform task management and configuration parameter management, and improve the flexibility of the system to achieve hierarchical management.

\subsection{Functional Design.}

According to the analysis of the current national network e-bank mobile Internet service, the user's main business and traffic accounted for a large proportion of power-related information browsing services, power business processing services and power purchase services, etc., for the current stateowned electronic channel mobile Internet services, user perception The application monitoring model should include the following three functions for user-aware detection and feedback.

(1) Business function

Initiating end-to-end testing of the application class service, by requesting a URL, initiating a request for the URL at the application layer, performing packet capture, process analysis, time division of each stage of the request and response process, obtaining the setup request time, sending the request time, and accepting Indicators such as response time. The web-based business mainly monitors the first-packet delay and the page open delay, which are two key user-perceived key quality indicators.

(2) Business and service awareness feedback function

Add user-aware feedback function to the front-end application layer to conduct business and service-aware feedback evaluation, especially after special cases such as failures. Questionnaire surveys were conducted on user perception and satisfaction on a regular basis.

(3) User-aware data intelligent analysis function

Semantic big data analysis and data mining for unstructured data in user-perceived data, automatically and profoundly extract user demand preferences and brand product opinions in massive data; A user-aware indicator system is constructed for structured data in user-aware data, and a statistical model is used to analyze user-perceived elements that affect service quality. 


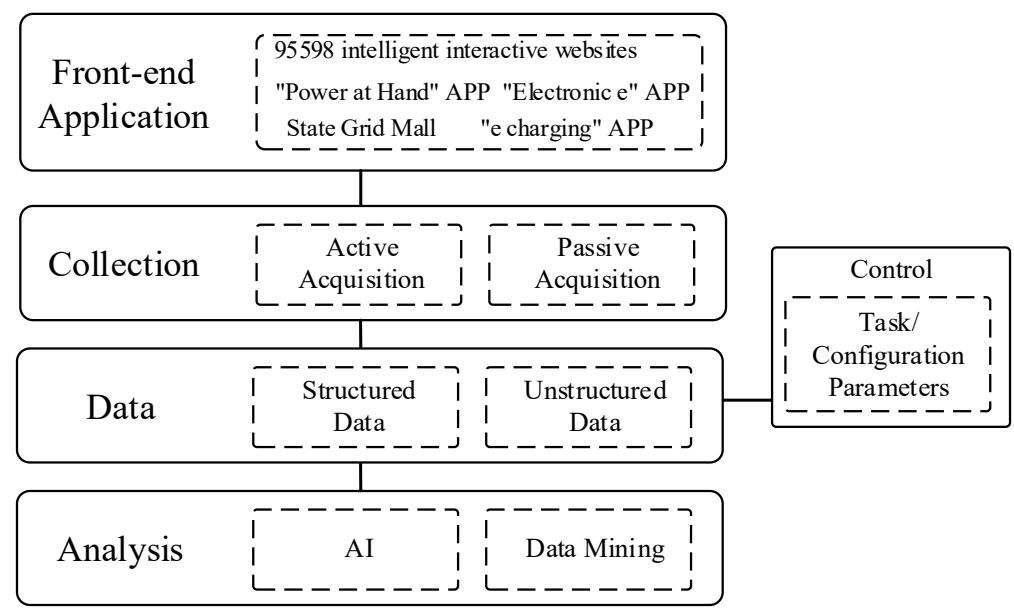

Fig 1.User-aware application supervision model

\section{Operation and Maintenance Supervision Model for Application Services}

With the development of intelligent and informationized power grids, various electronic channels of State Grid have become an indispensable supporting means to support the safe production and operation management of national power grids. It is especially important for the operation monitoring, daily operation and maintenance and fault handling of each electronic channel information system. The operation and maintenance management method from the traditional perspective of resource management can not monitor the operation of each channel application service, nor can it implement application fault location based on application topology and access path. It also cannot associate implement infrastructure and application services to automatic failure analysis. Therefore, the various electronic channels of the State Grid need to upgrade and integrate the traditional operation and maintenance monitoring mode, and establish an application-oriented service monitoring system, as shown in Figure 2.

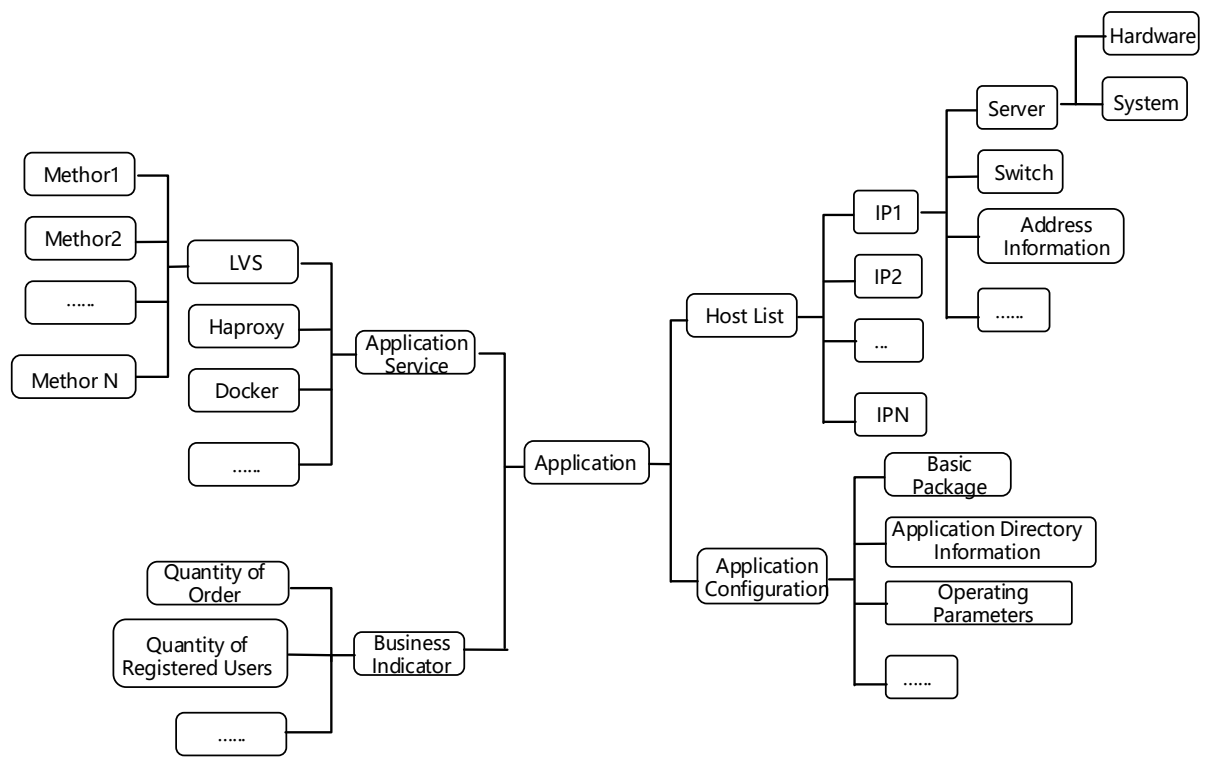

Fig 2. Application-oriented service monitoring model

In 2016, FORRESTER reported in the APM (Application Performance Management) report in the third quarter that with the increasing complexity of applications and technologies, the introduction of artificial intelligence will greatly reduce errors and manual manual errors. In the same year, Gartner listed six core APM core capabilities in the APM Core Competency Report, half of which were related to intelligence. Data and AI technology are the core of two intelligent operation and maintenance supervision models. The data is acquired by the monitoring system for application services. The AI 
model is trained through historical data and monitoring data to realize the intelligent operation and maintenance supervision model [5].

\subsection{Functional Design.}

The application-oriented service monitoring system effectively demonstrates the dependencies between applications and applications, applications and IT resources, and meets the requirements of modern business service management (BSM). The real-time monitoring of various technical indicators is the basis of operation and maintenance state analysis, fault prediction, and root cause analysis,shown as Table 1.

Table 1.Monitoring Description Table

\begin{tabular}{|c|c|}
\hline $\begin{array}{c}\text { Monitoring } \\
\text { Type }\end{array}$ & Indicator Type \\
\hline $\begin{array}{l}\text { Hardware } \\
\text { monitoring }\end{array}$ & Hardware temperature, fan speed, hardware voltage, etc. \\
\hline $\begin{array}{l}\text { System } \\
\text { resource } \\
\text { monitoring }\end{array}$ & $\begin{array}{l}\text { CPU (CPU usage percentage, user mode usage percentage, kernel state usage percentage, } \\
\text { CPU load, CPU running queue number, etc.), disk (disk read/write throughput, disk } \\
\text { read/write times, etc.), memory (memory usage, memory) Remaining amount, etc.), } \\
\text { network (network card out/in bandwidth, network card out/in packet amount, TCP status } \\
\text { monitoring, etc.), process (process port monitoring), etc. }\end{array}$ \\
\hline $\begin{array}{l}\text { Performance } \\
\text { monitoring }\end{array}$ & $\begin{array}{l}\text { Port (port existence, etc.), process (process presence, etc.), network traffic (sum of all } \\
\text { traffic, etc.), registration (registration response time, etc.), login (login response time, etc.), } \\
\text { recharge (recharge response time, etc.) }\end{array}$ \\
\hline $\begin{array}{l}\text { Business } \\
\text { monitoring }\end{array}$ & $\begin{array}{l}\text { Number of orders (per minute, daily, monthly, etc.), number of registered users (per } \\
\text { minute, daily, monthly, etc.), number of active users (daily, monthly, yearly, etc.), number } \\
\text { of users introduced by promotion activities, traffic introduced by promotion activities } \\
\text { Number, promotion activities, profit, etc. }\end{array}$ \\
\hline $\begin{array}{l}\text { Application } \\
\text { service } \\
\text { monitoring }\end{array}$ & LVS,Haproxy,Docker,Nginx,PHP,Memcached,Redis,Database,Rabbitmq ,etc. \\
\hline
\end{tabular}

\subsection{Supervision Technology}

Inter-application dependency analysis: By automatically analyzing the interdependencies between applications, graphical representations of application dependencies are used to clearly describe the dependencies between applications. It can help operators to quickly and accurately identify problems, locate problems, and help identify the root cause of the problem.

Natural language man-machine dialogue: Through the natural language analysis technology, construct an efficient human-machine dialogue interface, and use the information in the knowledge base to help the operation and maintenance personnel solve problems quickly through the "humanmachine dialogue" form.

Intelligent supervision technology: build intelligent dynamic operation and maintenance knowledge base through intelligent predictive analysis algorithm, historical problem processing plan, historical operation and maintenance experience base, intelligent monitoring result, use artificial intelligence technology to realize early detection and early detection of fault, and automatically provide corresponding processing suggestion.

Platform development model: build artificial intelligence operation and maintenance platform, connect various equipments in the business chain with artificial intelligence operation and maintenance platform, and build a intelligent operation and maintenance ecosystem.

\section{Summary}

In daily life, the consumption habits of the vast number of state-owned customers are changing with the development of the society. From the past, they must go to the business hall to now they can go to work without leaving their homes. The various electronic channels of the State Grid have greatly satisfied the needs of customers, and are the external image window of the State Grid. It is also an important way for the entire society to test and feel the achievements of smart grid construction. Through the user-aware application supervision model and the application-oriented service operation 
and maintenance supervision model, the customer experience and the business itself are fully supervised. With the aid of AI technology, the quality of the electronic channels of the national network is improved from the user's perspective, and the user's use satisfaction is improved.

\section{Acknowledgments}

This work is supported by the State Grid Science and Technology Project "Based on the "Internet +" Marketing Service Electronic Channel Unified Identity Authentication and Key Technologies for Service Supervision" Funding, Project No.: 52110417001D.

\section{References}

[1]. Lu Weilun. Research and application of information support for intelligent electronic service channels [A]. China Power Enterprise Association Science and Technology Development Service Center. Power Industry Informatization Excellent Papers 2014 - 2014 National Power Industry Integration Winning Conference and National Electric Power Enterprise Informatization Conference Award Paper [C]. China Power Enterprise Association Science and Technology Development Service Center:, 2014:7.

[2]. Qiu Fang. Artificial Intelligence to help data center intelligent operation and maintenance [N]. China Urban and Rural Financial News, 2016-04-06 (A03). Yang Keji, Han Li'an. Pile Foundation Engineering [M]. Beijing: People's Communications Publishing House, 1992.

[3]. Cao Weihua, Xu Yuting, He Xiaodong, Li Wenyun. Research and deployment of mobile Internet service perception APP system[J]. Guangdong Communications Technology, 2015, 35(11): 2-5. [2017-09-09].

[4]. Du Wei. Research on Key Technologies of Service Quality in Heterogeneous Converged Network for Customer Perception [D]. Beijing University of Posts and Telecommunications, 2015.

[5]. Lei Jun. Advantages and recommendations of intelligent monitoring technology in telecom enterprise operation and maintenance support system [J/OL]. Electronic Technology and Software Engineering, 2017, (12): 155. (2017-06-23)[2017- 09-09]. http:// kns. cnki.net/ kcms/ detail/10.1108.TP.20170623.1140.240.html. 